\title{
An estimate of the structural parameters of the Large Magellanic Cloud using red clump stars
}

\author{
S. Subramanian ${ }^{1,2}$ and A. Subramaniam ${ }^{1}$ \\ 1 Indian Institute of Astrophysics, Koramangala II Block, 560034 Bangalore, India \\ e-mail: [smitha;purni] @iiap.res.in \\ 2 Department of Physics, Calicut University, Calicut, Kerala, India
}

Received 4 February 2010 / Accepted 12 June 2010

ABSTRACT

\begin{abstract}
Aims. We used the red clump stars from the VI photometric data of the Optical Gravitational Lensing Experiment (OGLE III) survey and from the Magellanic Cloud Photometric Survey (MCPS) to estimate the structural parameters of the LMC disk, such as the inclination, $i$ and the position angle of the line of nodes $\left(\mathrm{PA}_{\text {lon }}\right), \phi$.

Methods. The observed disk region is divided into sub-regions. The dereddened peak $I$ magnitude of the red clump stars in each sub-region is used to obtain the relative distances and hence the $z$ coordinate. The RA and Dec of each sub-region is converted into $x$ and $y$ cartesian coordinates. A weighted least-square plane-fitting method is applied to these $x, y, z$ data to estimate the structural parameters of the LMC disk.

Results. We find an inclination of $i=23^{\circ} .0 \pm 0.8$ and $\mathrm{PA}_{\mathrm{lon}}, \phi=163^{\circ} .7 \pm 1.5$ for the LMC disk using the OGLE III data and an inclination of $i=37.4 \pm 2.3$ and $\mathrm{PA}_{\mathrm{lon}} \phi=141^{\circ} .2 \pm 3.7$ for the LMC disk using the MCPS data. Extra-planar features, which are in front as well as behind the fitted plane, are seen in both the data sets.

Conclusions. Our estimates of the inclination and position angle of the line of nodes are comparable with some of the previous estimates. The effect of choice of center, reddening, and area covered on the estimated parameters are discussed. Regions in the northwest, southwest and southeast of the LMC disk are warped with respect to the fitted plane. We also identify a symmetric but off-centered warp in the inner LMC. We identify that the structure of the LMC disk inside the 3 degree radius is different from the outside disk in a way that the inner LMC has relatively less inclination and relatively large $\mathrm{PA}_{\text {lon. }}$. The 3D plot of the LMC disk suggests an off-centered increase in the inclination for the northeastern regions, which might be due to tidal effects. We suggest that the variation in the planar parameters estimated by various authors as well as in this study is caused by the difference in coverage and the complicated inner structure of the LMC disk. In the inner LMC, the stellar and the HI disk are found to have similar properties.
\end{abstract}

Key words. Magellanic Clouds - galaxies: structure - stars: horizontal-branch

\section{Introduction}

The Large Magellanic Cloud (LMC) is one of our nearest neighbors and is located at a distance of around $50 \mathrm{kpc}$. Magellanic Clouds (MCs) were believed to have had interactions with our Galaxy as well as between each other (Westerlund 1997). It is also believed that the tidal forces due to these interactions have caused structural changes in this galaxy. The study of the LMC's structure is important to understand the effect of interactions in this galaxy.

The LMC is believed to be a disk galaxy with planar geometry, and the orientation measurements of the LMC disk plane have been done previously by various authors using different tracers. By assuming the LMC disk to be circular when viewed face-on, de Vaucouleurs \& Freeman (1972) found a disk inclination of $i=27^{\circ} \pm 2^{\circ}$ and a position angle of the line of nodes $\left(\mathrm{PA}_{\mathrm{lon}}\right), \phi=170^{\circ} \pm 5^{\circ}$ from the elliptical outer isophotes in red exposures. Based on the analysis of spatial variations in the apparent magnitude of asymptotic giant branch (AGB) stars in the near-IR colour-magnitude diagrams extracted from the Deep Near-Infrared Southern Sky Survey (DENIS) and Two Micron All-Sky Survey (2MASS), van der Marel \& Cioni (2001) estimated an $i=34.7 \pm 6.2$ and $\phi=122.5 \pm 8.3$ for the LMC disk between 2.5 to 6.7 from the LMC center.
Olsen \& Salyk (2002) derived an $i=35^{\circ} .8 \pm 2^{\circ} .4$ and $\phi=145^{\circ} \pm 4^{\circ}$ by studying the red clump (RC) magnitudes in the inner LMC, excluding the bar region. They also showed that the southwestern part of the LMC disk is warped. Recently, Koerwer (2009) derived an $i=23^{\circ} .5 \pm 0^{\circ} .4$ and $\phi=154^{\circ} .6 \pm 1^{\circ} .2$, using the $J H$ photometric data of RC stars from the Infrared Survey Facility (IRSF) Magellanic Clouds Point Source Catalogue. Cepheids are also used to obtain the orientation measurements of the LMC disk. Caldwell \& Coulson (1986) analyzed a sample of 73 cepheids and obtained $i=28^{\circ} .6 \pm 5^{\circ} .9$ and $\phi=142^{\circ} .4 \pm 7.7$. Nikolaev et al. (2004) based on a sample of 2000 MACHO cepheids obtained an $i=30^{\circ} .7 \pm 1^{\circ} .1$ and $\phi=151^{\circ} \pm 2^{\circ} .4$. Persson et al. (2004) obtained an $i=27^{\circ} \pm 6^{\circ}$ and $\phi=127^{\circ} \pm 10^{\circ}$ from the analysis of 92 near infrared light curves of Cepheids. Various studies of HI gas estimated the structural parameters of the HI disk of the LMC. Feitzinger et al. (1977) derived an inclination of $33^{\circ} \pm 3^{\circ}$ and $\mathrm{PA}_{\text {lon }}$ of $168^{\circ} \pm 4^{\circ}$ by geometrical means. The HI velocity studies by Luks \& Rohlfs (1992) revealed two kinematic components, the L (lower velocity) component and the $\mathrm{D}$ (disk) componenet. The $\mathrm{PA}_{\mathrm{lon}}$ of around $162^{\circ}$ was estimated for the disk component. Kim et al. (1998) estimated the $\mathrm{PA}_{\text {lon }}$ of $\mathrm{HI}$ disk to be around $168^{\circ}$ and an inclination of $22^{\circ} \pm 6^{\circ}$.

The red Clump (RC) stars are core helium burning stars, which are the metal-rich and slightly more massive counter parts 
of the horizontal branch stars. They have a tightly defined color and magnitude, and appear as an easily identifiable component in the color-magnitude diagrams (CMDs). RC stars were used as standard candles for distance determination by Stanek et al. (1998). They used the intrinsic luminosity to determine the distance to the LMC. Subramaniam (2003) used the constant magnitude of RC stars to show that the LMC has structures and warps in the bar region. Their characteristic color was used by Subramaniam (2005b) to estimate the reddening map towards the central LMC. Subramanian \& Subramaniam (2009) estimated the depth of the Magellanic Clouds using the dispersions in the magnitude and color distributions of RC stars. As mentioned in the earlier paragraph, $\mathrm{RC}$ stars are also used to estimate the orientation measurements of the LMC disk plane.

In this paper we use the photometric data of the RC stars in the $V$ and $I$ pass bands from the Magellanic Cloud Photometric Survey (MCPS) and the Optical Gravitational Lensing Experiment (OGLE III) to estimate the structural parameters of the LMC disk plane. These catalogs have homogeneous and continuous sampling of stars spread over the inner LMC up to a radius of about 6 degrees. Olsen \& Salyk (2002) studied the structure of the LMC using RC stars in discrete and widely separated pointings located away from the bar region. Koerwer (2009) used $J H$ photometric data of RC stars in the inner region of the LMC to study the structure, where the sample has contamination from AGB stars. Compared to the above two studies, we use a homogeneous and continuous sample of RC stars, with minimal contamination from stars in other evolutionary stages.

The plan of this paper is as follows. Data sources are explained in the next section. In Sect. 3 we give details of the analysis. Results are given in Sect. 4 and their implications are discussed in Sect. 5, followed by conclusions in Sect. 6.

\section{Data}

The OGLE III survey (Udalski et al. 2008) presented VI photometry of $40 \mathrm{deg}^{2}$ of the LMC consisting of about 35 million stars. We divided the observed region into 1854 regions (with a reasonable number of RC stars, 500-9000) with a bin size of $8.88 \times 8.88 \mathrm{arcmin}^{2}$. Regions with RC stars in the range $500-700$ are located in the eastern and western ends of the disk region covered by the OGLE III. The average photometric error of RC stars in $I$ and $V$ bands are around 0.05 mag. Photometric data with errors less than $0.15 \mathrm{mag}$ are considered for the analysis. For each sub-region the $(V-I)$ vs. $I$ CMD is plotted and RC stars are identified. A sample CMD is shown in the upper left panel of Fig. 1. For all the regions, RC stars are well within the box of CMD, with boundaries $0.65-1.35 \mathrm{mag}$ in $(V-I)$ colour and 17.5-19.5 mag in I magnitude.

The Magellanic Cloud Photometric Survey (MCPS, Zaritsky et al. 2004) of the central 64 square degrees of the LMC obtained photometric data of around 24 million stars in the $U, B, V$, and $I$ pass bands. Data with errors in the $V$ and $I$ pass bands less than $0.15 \mathrm{mag}$ are taken for the analysis. The regions away from the bar are less dense compared to the bar region. The total observed regions are divided into 1512 sub-regions each with an area of approximately $10.53 \times 15 \mathrm{arcmin}^{2}$. Out of 1512 regions only 1377 regions have a reasonable number of RC stars (100-2000) to do the analysis. $(V-I)$ vs. I CMDs for each region are plotted, and RC stars are identified as described above.
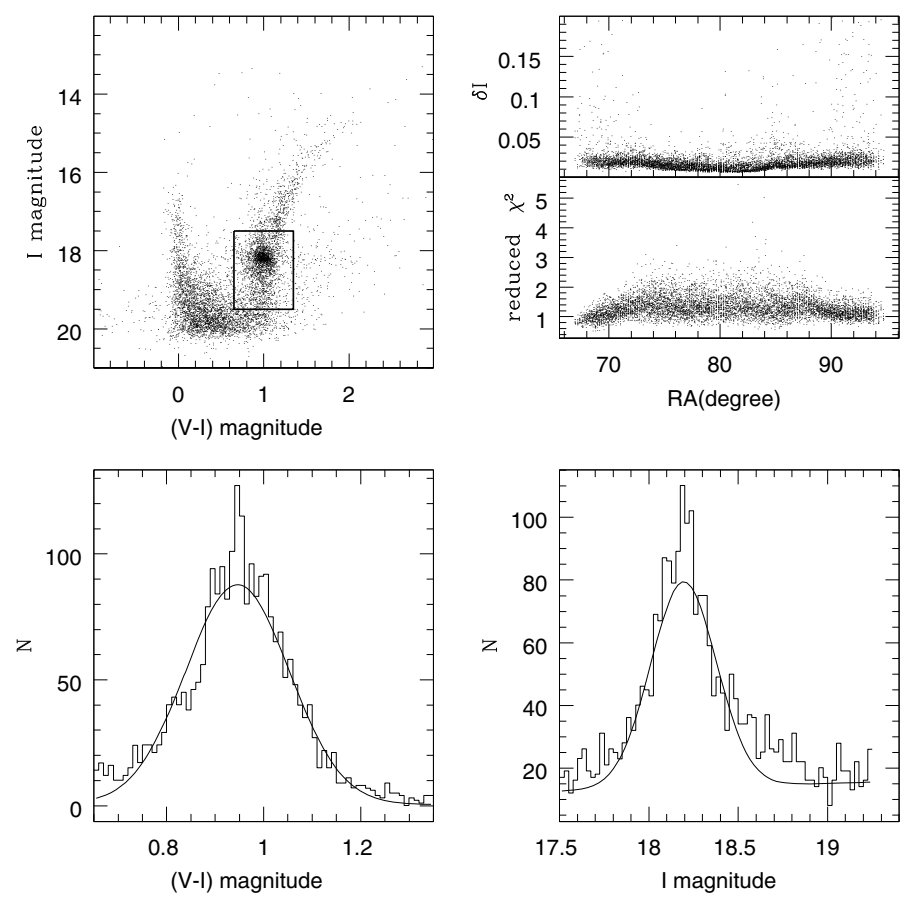

Fig. 1. Color magnitude diagram of an LMC OGLE III region is shown in the upper left panel. The box used to identify the RC population is also shown. Typical color (lower left panel) and magnitude (lower right panel) distribution of Red Clump stars are shown. The best fit to the distributions are also shown. The reduced $\chi^{2}$ of the magnitude distribution and the fit error of the peak $I$ magnitude as a function of RA are shown in the right middle panel and right top panel respectively.

\section{Analysis}

The dereddened peak RC magnitude is used to obtain the structural parameters of the LMC disk. RC stars occupy a compact region in the CMDs and they have a constant characteristic $I$ band magnitude and $(V-I)$ color. Their number distribution profiles resemble a Gaussian. The peak values of their color and magnitude distributions are used to obtain the dereddened RC magnitude and hence the structural parameters of the LMC disk. The analysis is similar to that done by Olsen \& Salyk (2002).

To obtain the number distribution of the RC stars, they are binned in both color and magnitude with a bin size of 0.01 and $0.025 \mathrm{mag}$, respectively. These distributions are fitted with a Gaussian + Quadratic polynomial. The Gaussian represents the $\mathrm{RC}$ stars and the other terms represent the red giants in the region. A nonlinear least-square method is used for fitting and the parameters are obtained. In the lower two panels of Fig. 1, the distributions as well as the fitted curves for an LMC OGLE III region are shown. The left lower panel is the color distribution and right panel is the magnitude distribution. The parameters obtained are the coefficients of each term in the function used to fit the profile, error in the estimate of each parameter, and reduced $\chi^{2}$ value. For both data sets we estimated the peaks in $I$ mag and $(V-I)$ mag of the distributions, associated errors with the parameters, and goodness of fit.

The reduced $\chi^{2}$ values and the fit-error of the peak of the I magnitude distribution are plotted against RA in the right middle panel and right upper panel of Fig. 1 respectively. Regions with peak errors greater than 0.1 mag and those with reduced $\chi^{2}$ value greater than 2.0 are omitted from the analysis. Thus the regions used for final analysis became 1262 for the OGLE III data and 1231 for the MCPS data. 
The peak values of the color, $(V-I)$ mag at each location is used to estimate the reddening. The reddening is calculated using the relation $E(V-I)=(V-I)_{\mathrm{obs}}-0.92 \mathrm{mag}$. The intrinsic color of the RC stars is taken to be 0.92 mag (Olsen \& Salyk 2002). The reddening values were found to be negative for 451 locations in the MCPS data set. These regions were mostly located near the center. Zaritsky et al. (2004) found many regions with negative $A_{\mathrm{v}}$ values while estimating the extinction towards the LMC using the MCPS data and assigned an extinction of zero for those regions. They suggested that the negative extinction values are due to observational uncertainties. Reddening plays an important role in the estimation of peak magnitude and hence the estimated structure, thus the regions which showed negative values for reddening estimates are omitted from our analysis. Hence the number of regions used for final analysis became 780 for the MCPS data. Assigning a zero reddening to those regions with negative reddening estimates and its impact on the estimates of the LMC disk parameters are discussed in Sect. 5. The selection of the intrinsic $(V-I)$ color of the RC stars may also be the reason for obtaining negative reddening values. For the MCPS data, the intrinsic $(V-I)$ color of the RC stars can be set to produce the median reddening obtained by Schlegel et al. (1998) towards the LMC. Implications of resetting the intrinsic $(V-I)$ color of RC stars for the MCPS data are discussed in detail in Sect. 5. Negative reddening was not found for any regions in the OGLE III data.

The interstellar extinction is estimated by $A_{I}=1.4 \times E(V-I)$ (Olsen \& Salyk 2002). After correcting the mean $I$ mag for interstellar extinction, $I_{0}$ for each region is estimated. The difference in $I_{0}$ between regions is a measure of the relative distances, so that $0.1 \mathrm{mag}$ in $\Delta I$ corresponds to $2.3 \mathrm{kpc}$ in distance. The variation in $I_{0}$ mag is converted into relative distances. The relative distance is

$\Delta D=\left(I_{0}\right.$ mean $-I_{0}$ of each region $) \times 23 \mathrm{kpc}$.

The error in $I_{0}$ is estimated as

$\delta I_{0}^{2}=(\operatorname{avg} \text { error in peak } I)^{2}+(\operatorname{avg} \text { err in peak }(V-I))^{2}$.

Here, the variation in $I_{0}$ is considered only due to the line of sight distance variation within the galaxy. The error in magnitude is also converted into error in distance.

In this analysis, we have not incorporated the incompleteness due to crowding, especially in the central regions where the effect is expected to be prominent. In order to estimate the effect due to crowding and the incompleteness, we compared the $I_{0}$ values with and without incompleteness correction (Subramanian \& Subramaniam 2009). Subramanian \& Subramaniam (2009) used the OGLE II data for the analysis. They did not find any significant difference between the parameters, suggesting that the incompleteness/crowding does not affect the results presented here. Subramaniam \& Subramanian (2009) also compared the $I_{0}$ values obtained from the OGLE III with the $I_{0}$ values obtained from the OGLE II, where incompleteness correction was incorporated. They did not find any significant change in the $I_{0}$ values.

The relative distance of each region of the LMC is obtained from the variation in the $I_{0}$ magnitude. Then the $x, y$, and $z$ coordinates are obtained using the transformation equations given below (van der Marel \& Cioni 2001; see also Appendix A of Weinberg \& Nikolaev 2001)

$x=-D \sin \left(\alpha-\alpha_{0}\right) \cos \delta$,

$y=D \sin \delta \cos \delta_{0}-D \sin \delta_{0} \cos \left(\alpha-\alpha_{0}\right) \cos \delta$,

$z=D_{0}-D \sin \delta \sin \delta_{0}-D \cos \delta_{0} \cos \left(\alpha-\alpha_{0}\right) \cos \delta$, where $D_{0}$ is the distance to the center of the LMC and $D$, the distance to the each sub-region is given by $D=D_{0}+\Delta D$. The $(\alpha, \delta)$ and $\left(\alpha_{0}, \delta_{0}\right)$ represents the RA and Dec of the region and the center of the LMC respectively. In our analysis, the optical center of the LMC, $05^{\mathrm{h}} 19^{\mathrm{m}} 38^{\mathrm{s}} .0-69^{\circ} 27^{\prime} 5^{\prime \prime} .2$ (J2000) (de Vaucouleurs \& Freeman 1972) is taken as the center of the LMC.

Once we have the $x, y$, and $z$ coordinates we can apply a weighted least square plane fit to obtain the structural parameters of the LMC disk. The equation of the plane used for the plane fit is given by

$A x+B y+C z+D=0$, with the constraint $A^{2}+\mathrm{B}^{2}+\mathrm{C}^{2}=1$.

From the coefficients of the plane $A, B$ and $C$, the inclination, $i$ and the position angle of line of nodes $\left(\mathrm{PA}_{\text {lon }}\right), \phi$ can be calculated using the formula given below

Inclination, $i=\arccos (C)$

$\mathrm{PA}_{\text {lon }}, \phi=\arctan (-A / B)-\operatorname{sign}(B) \pi / 2$.

A weighted least-square plane fit is applied to the $x, y$, and $z$ data of the MCPS \& OGLE III data sets. The inclination, $i$ and the $\mathrm{PA}_{\text {lon }}$ of the LMC disk are estimated from the plane-fitting procedure.

We calculated the deviations of the LMC disk from the plane with estimated coefficients. The expected $z$ for a plane is calculated with the equation of a plane, $A x+B y+C z+D=0$. The difference in the expected and calculated $z$ values is taken as the deviation of the LMC disk from the plane. Thus the extra-planar features of the LMC disk are identified and quantified. Once the deviations are estimated, the regions with deviations above three times the error in $z$ are omitted and the plane-fitting procedure is applied to the remaining regions to re-estimate the structural parameters of the LMC disk plane.

The error in the estimate of the LMC disk parameters is calculated from the error associated with the $z$ values. The planefitting procedure is repeated with the positive and negative deviation of $z$ values and the LMC disk parameters are calculated again. Thus the range of structural parameters are estimated, which are converted as the error in the estimate of the parameters.

\section{Results}

The structural parameters of the LMC disk are estimated using the dereddened mean magnitude of RC stars. The MCPS and OGLE III data sets are used for the estimate. A weighted planefitting procedure applied to 780 regions of the MCPS data set gives an inclination of $i=38.2 \pm 5^{\circ} .0 \& \mathrm{PA}_{\mathrm{lon}}, \phi=141^{\circ} \pm 9.8$ for the LMC disk. The deviation of the LMC disk regions from the estimated plane are calculated as explained in the previous section. The average error in the estimate of $I_{0}$ mag is converted into distance and its around $500 \mathrm{pc}$ for the MCPS data. Deviations above 3 sigma are considered as significant deviations from the fitted plane. Figure 2 shows the deviation of the MCPS regions from the plane. In the bottom panel, all the regions used for the analysis are plotted. The black points are those which are on the fitted plane, red points are disk regions which are behind the plane and blue points are the disk regions which are in front of the plane. In the upper panel only the regions with deviations above 3 sigma are plotted and the size of the points are proportional to the amplitude of the deviation. Here also, red points are regions behind the fitted plane and blue are in front of the 

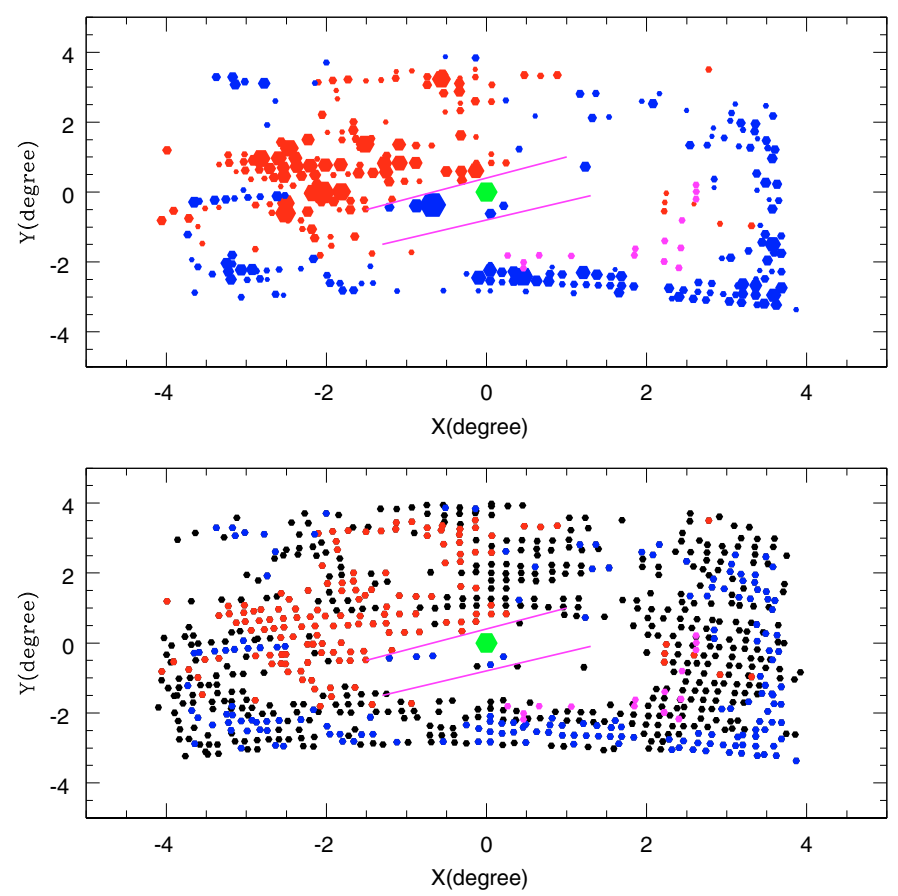

Fig. 2. In the lower panel, black dots represent the regions on the fitted plane, red dots represent the regions behind the fitted plane and the blue dots represent the regions which are in front of the fitted plane. The upper panel shows only the regions with deviations greater than 3 sigma from the LMC disk plane. The size of the points are proportional to the amplitude of the deviations. Magenta dots in both panels are the regions which are suggested as warps by Olsen \& Salyk (2002). The green hexagon in both plots represents the optical center of the LMC.

fitted plane. From the plots we can see that there are many regions which deviate from the planar structure of the LMC disk. The plot shows that the RC stars in the regions southeast, southwest and northwest of the LMC disk are brighter than what is expected from the plane fit. Also, some regions northeast to the LMC bar are dimmer than expected.

The RC stars in the LMC disk are a heterogeneous population, and therefore they would have a range in mass, age, and metallicity. The density of stars in various locations will also vary with the local star-formation rate as a function of time. These factors result in a range of magnitude and color of the net population of RC stars in any given location and would contribute to the observed peaks in magnitude and color distributions. Therefore, the deviations found in some regions may also be due to these population differences of RC stars. Then the brightening of RC stars in the southeast, southwest and northwest of the LMC disk indicates either a different RC population and/or these regions are warped. Similarly, the dimming of $\mathrm{RC}$ stars in the north east of the LMC bar indicates a difference in RC population and/or these regions are behind the fitted plane. Based on the studies of the LMC clusters, Grocholski et al. (2006) found that the LMC lacks the metallicity gradient typically seen in the galaxies. Studies by Subramaniam \& Anupama (2002), Olsen \& Salyk (2002) and van der Marel \& Cioni (2001) found no noticeable change in age and metallicity of the RC population in the central region of the LMC. As the regions we study are located in the central region of the LMC, the effects of population difference of RC stars in these regions are likely to be negligible. Hence we can see from these plots that the southeast, southwest and the northwestern regions of the
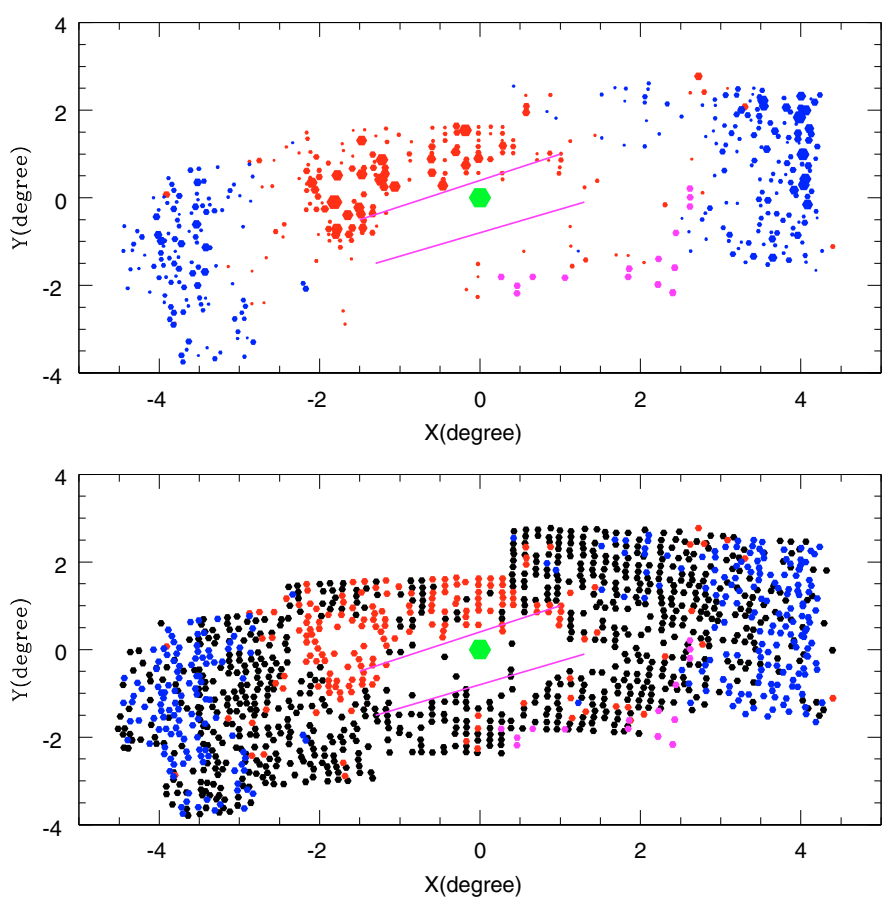

Fig. 3. In the lower panel, black dots represent the regions on the fitted LMC plane, red dots represent the regions behind the fitted plane and the blue dots represent the regions which are in front of the fitted plane. The upper panel shows only the regions with deviations, greater than 3 sigma, from the LMC disk plane. The size of the points are proportional to the amplitude of the deviations. Magenta dots in both panels are the regions which are suggested as warps by Olsen \& Salyk (2002). The green hexagon in both plots represents the optical center of the LMC.

LMC disk may be warped and the regions northeast to the bar of the LMC are behind the fitted plane. In the figure, the regions suggested by Olsen \& Salyk (2002) as warps are over plotted as magenta points. Their points in the southwestern regions are near the warped regions suggested by us, though they do not coincide.

The extra-planar features seen in the LMC disk would have affected the estimate of the planar parameters of the LMC disk. The reduced $\chi^{2}$ value for the estimate of planar parameters using the MCPS data set is 2.0. The higher value of reduced $\chi^{2}$ value can be due to the presence of structures in the LMC disk. So we omitted the regions with deviations above 3 sigma and the planar parameters are estimated again. Around 320 regions out of 780 regions showed deviations above 3 sigma and the remaining 460 regions are used for the re-estimate. Thus the structural parameters obtained for the LMC disk plane after removing the extra planar features are inclination, $i=37.2 \pm 2.3$ and $\mathrm{PA}_{\text {lon }}, \phi=141^{\circ} .4 \pm 3^{\circ} .7$. The reduced $\chi^{2}$ value for the plane fitting in this case is 0.4 .

The plane-fitting procedure applied to 1262 regions of the OGLE III data gives an $i=21^{\circ} 0 \pm 2.2$ and $\phi=171^{\circ} .1 \pm 8.8$ for the LMC disk. As done for the MCPS data, deviations from the LMC disk plane with the above planar parameters are estimated. The average error in the estimate of $z$ distance for the OGLE III data set is around $300 \mathrm{pc}$. The regions which show deviations above 3 sigma are considered as real deviations. Figure 3 shows the deviation plot for the OGLE III data. The bottom panel shows all the 1262 regions used for plane fitting and the upper panel shows only those regions which show deviations above 3 sigma. 

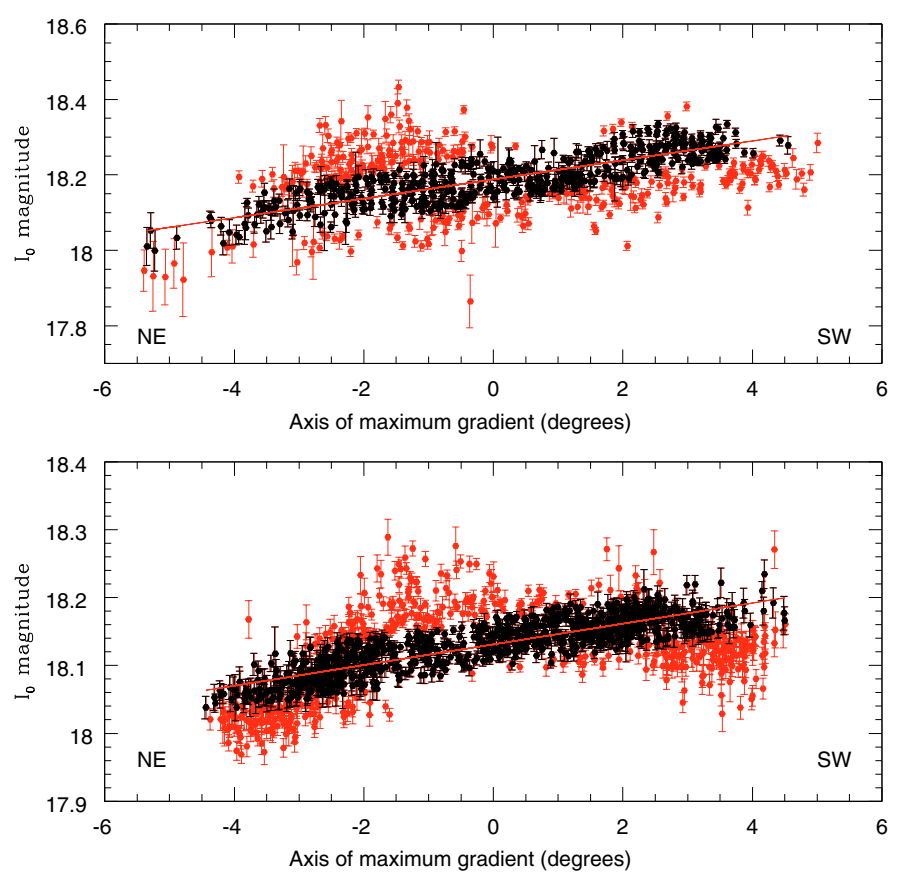

Fig. 4. Dereddened RC magnitude plotted against the axis of maximum gradient. The red points are regions which show deviation larger than 3 sigma. The direction of inclination is shown as red line. The upper panel is for the MCPS data and the lower panel is for the OGLE III.

In the upper panel the size of the points are proportional to the amplitude of the deviations. The color code in the figure is the same as in Fig. 2. As in the MCPS data, the OGLE III also shows, similar brightening in the southeast, northwest and southwestern regions of the LMC disk. The northeastern part adjacent to the LMC bar is also dimmer in this plot similar to the MCPS plot. So either the northwestern, south western and southeastern parts of the LMC disk regions are in front of the fitted plane and/or the RC population in these regions is different. Again the northeastern part north of the LMC bar is either behind and/or the $\mathrm{RC}$ population in this region is different. As done for the MCPS data set, the extra-planar features are removed and the parameters are estimated again for the OGLE III data set. Out of 1262 regions, 397 regions showed deviations above 3 sigma, and these regions are removed from the plane-fitting procedure. Thus the structural parameters obtained for the LMC disk plane after removing the extra planar features are inclination, $i=23^{\circ} \pm 0.8$ and $\phi=163.7 \pm 1.5$. The reduced $\chi^{2}$ value of the plane-fitting procedure with all the 1262 regions was 1.0 and now, when the region with large deviations are removed, the reduced $\chi^{2}$ became 0.3 .

After removing the extra-planar features, $\phi$ estimated for the MCPS and the OGLE III data sets are $141^{\circ} .2 \pm 3^{\circ} .7$ and $163^{\circ} .7 \pm 1^{\circ} .6$ respectively. The dereddened $\mathrm{RC}$ magnitude is plotted against the axis perpendicular to the line of nodes, axis of maximum gradient and its shown in Fig. 4. The upper panel shows the plot for the MCPS data and the lower panel shows the plot for the OGLE III data. From both plots we can clearly see the effect of inclination. The slopes estimated for both data sets excluding the regions with deviations above 3 sigma are $0.0254 \mathrm{mag} /$ degree, and the $y$-intercept is $18.18 \mathrm{mag}$ for the MCPS data and a slope of $0.0152 \mathrm{mag} /$ degree and the $y$-intercept of $18.13 \mathrm{mag}$ for the OGLE III. The inclination estimated for the MCPS data is 33.3 degrees and 21.5 degrees for the OGLE III. The inclinations estimated from the plots for both data sets match well with the inclination values estimated from the plane-fitting procedure for the same data set.

The $y$-intercepts obtained from Fig. 4 for both data sets are the mean $I_{0}$ value of RC stars. As RC stars are standard candles, this value is a measure of the distance to the center of the LMC. The $y$-intercepts obtained for the OGLE III and the MCPS data sets from Fig. 4 are $18.13 \pm 0.01 \mathrm{mag}$ and $18.18 \pm 0.01 \mathrm{mag}$ respectively. The distance modulus, $\mu_{0}$ to the LMC center can be estimated using the formula

$\mu_{0}=I_{0 \text { mean }}-M_{I(\mathrm{LMC})}$.

In the above equation, $M_{I(\mathrm{LMC})}$ is the $I$-band absolute magnitude of RC stars in the LMC. Stanek et al. (1998) used the absolute $I$-band magnitude of RC stars in the Hipparcos sample as the zero point for distance estimation to the LMC. Later Girardi \& Salaris (2001) found from their simulations of local clump and those found in the LMC that there is a systematic magnitude difference between them. This systematic magnitude difference is due to the differences in the age, metallicity, and star-formation rate of the RC stars in the Galaxy and the LMC. Girardi \& Salaris (2001) simulated the RC stars in the LMC bar as well as in the outer fields using the star-formation rate estimated by Holtzman et al. (1999) and the age-metallicity relation from Pagel \& Tautvaisiene (1998). From their simulations, they estimated a $M_{I(\mathrm{LMC})}$ of -0.371 for the central region of the LMC bar. Thus the distance moduli, $\mu_{0}$ to the LMC center estimated from the OGLE III and the MCPS data are 18.50 \pm 0.01 and $18.55 \pm 0.01$ respectively after correcting for population effects. These values agrees well with the previous estimates of $18.5 \pm$ 0.02 (Alves 2004) and $18.53 \pm 0.07$ (Salaris \& Girardi 2002) toward the LMC.

\section{Discussion}

The dereddened peak I magnitude of RC stars from the OGLE III and the MCPS data sets are used to estimate the structural parameters of the LMC disk and hence the deviations of the LMC regions from the plane. The planar parameters of the LMC disk obtained from the analysis of 1262 regions of the OGLE III data turned out to be an inclination of $i=23^{\circ} \pm 0.9$ and $\mathrm{PA}_{\mathrm{lon}}$, $\phi=163.7 \pm 1$. 6 . From the analysis of 780 regions of the MCPS data, an inclination, $i=37.4 \pm 2.5$ and $\mathrm{PA}_{\text {lon }} \phi=141.2 \pm 3.7$ are obtained. Previously many studies have been done to obtain the planar parameters of the LMC disk using various tracers. The values obtained from those studies along with our estimates are summarised in Table 1. Tracers used in those studies are also mentioned in the table. Our estimates based on the analysis of the MCPS data is matching well within the error bars with the estimates of Olsen \& Salyk (2002). Various studies of the LMC disk and bar regions (Fig. 6 given in Koerwer 2009; Fig. 2 in Subramaniam \& Subramanian 2009; and Fig. 4 in Subramaniam 2003) have shown that it is a highly structured galaxy. The estimate of the planar parameters are likely to be severely affected by these structures. The difference in the regions, coverage of the LMC, and the tracer used for the estimate of the planar parameters may be the reason for the differences in the estimated parameters of various studies. Also, Nikolaev et al. (2004) showed that the analysis based on the photometric data from the concentric rings in the inner LMC is strongly dependent on the adopted LMC center, which can cause a variation of about 35 degrees in the values of $\mathrm{PA}_{\text {lon }}$. 
Table 1. Summary of orientation measurements of LMC disk plane.

\begin{tabular}{lrrc}
\hline \hline Reference & Inclination, $i$ & \multicolumn{1}{c}{$\mathrm{PA}_{\text {lon }}, \phi$} & Tracer used for the estimate \\
\hline de Vaucouleurs \& Freeman (1972) & $27^{\circ} \pm 2^{\circ}$ & $170^{\circ} \pm 5$ & Isophotes \\
Feitzinger et al. (1977) & $33^{\circ} 0 \pm 3^{\circ}$ & $168^{\circ} \pm 4^{\circ}$ & HI \\
Caldwell \& Coulson (1986) & $28^{\circ} .0 \pm 5^{\circ} .9$ & $142^{\circ} .4 \pm 7^{\circ} .7$ & Cepheids \\
Luks \& Rohlfs (1992) & - & $162^{\circ} .0$ & HI \\
Kim et al. (1998) & $22^{\circ} 0 \pm 6^{\circ}$ & $168^{\circ} .0$ & HI \\
van der Marel \& Cioni (2001) & $34^{\circ} .7 \pm 6^{\circ} .2$ & $122^{\circ} .5 \pm 8^{\circ} .3$ & AGB stars \\
Olsen \& Salyk (2002) & $35^{\circ} .8 \pm 2^{\circ} .4$ & $145^{\circ} \pm 4^{\circ}$ & Red clump stars \\
Nikolaev et al. (2004) & $30^{\circ} 7 \pm 1^{\circ} .1$ & $151^{\circ} \pm 2^{\circ} .4$ & Cepheids \\
Persson et al. (2004) & $27^{\circ} .0 \pm 6^{\circ} .0$ & $127^{\circ} \pm 10^{\circ} .0$ & Cepheids \\
Koerwer (2009) & $523^{\circ} \pm 0.4$ & $154^{\circ} .6 \pm 1.2$ & Red clump stars \\
\hline Our estimates & & & \\
OGLE III & $23^{\circ} \pm 0.8$ & $163^{\circ} .7 \pm 1^{\circ} .5$ & Red clump stars \\
MCPS & $37^{\circ} 4 \pm 2^{\circ} .3$ & $141^{\circ} .2 \pm 3^{\circ} .7$ & Red clump stars \\
\hline
\end{tabular}

\subsection{Choice of the LMC center}

The adopted center of the LMC for the analysis may have an effect on the estimated parameters. We used the plane fitting method, rather than the ring analysis, which is probably less affected by the choice of the center. For our analysis, we have taken the optical center, $05^{\mathrm{h}} 19^{\mathrm{m}} 38^{\mathrm{s}} .0-69^{\circ} 27^{\prime} 5^{\prime \prime} .2$ (J2000) (de Vaucouleurs \& Freeman 1972), as the center of the LMC. To see the effect of the adopted center on the estimated parameters, we did the analysis with the HI rotation center (Kim et al. 1998), geometric center of the sample Cepheids used in the analysis of Nikolaev et al. (2004) and also the carbon stars isopleths center (van der Marel \& Cioni 2001) as the center of the LMC. We find that the choice of center causes only marginal changes in the estimated parameters, and the changes are within the error. The estimated parameters with different centers are given in Table 2.

\subsection{Choice of area of the regions}

In order to check the effect of area of the bins used in our analysis, the OGLE III region is divided into sub regions with area, $8.88 \times 16.76 \mathrm{arcmin}^{2}$, comparable to the area of sub-region of the MCPS data. The whole analysis is repeated and the structural parameters of the LMC disk are estimated. The parameters, inclination and $\mathrm{PA}_{\text {lon }}$ obtained are $24.5^{\circ} \pm 0.5$ and $166^{\circ} \pm 1^{\circ}$ respectively. These values are comparable with our results obtained from the analysis of the OGLE III data with smaller area $\left(8.88 \times 8.88 \mathrm{arcmin}^{2}\right)$ sub-regions. This means that extending the area of sub-regions is unlikely to change the estimated parameters. But when the area of the OGLE III sub-region is made $4.44 \times 4.44 \mathrm{arcmin}^{2}$, smaller than $8.88 \times 8.88 \mathrm{arcmin}^{2}$, used for our analysis, there was problem in the plane-fitting procedure due to the large number of points. This can be due to the finer structures present in the inner LMC.

\subsection{Effect of reddening}

The other important point to be discussed is the role of reddening in the estimate of the structure of the LMC disk. The extra-planar features which are found both behind the disk and in front of the disk could be in the plane of the LMC disk itself if there were an over-estimate or under-estimate of the reddening. It has been demonstrated by Zaritsky et al. (1997) that the extinction property of the LMC varies both spatially and as a function of stellar population. In our study, the dereddening of RC stars is done using the reddening values estimated from the RC stars itself.
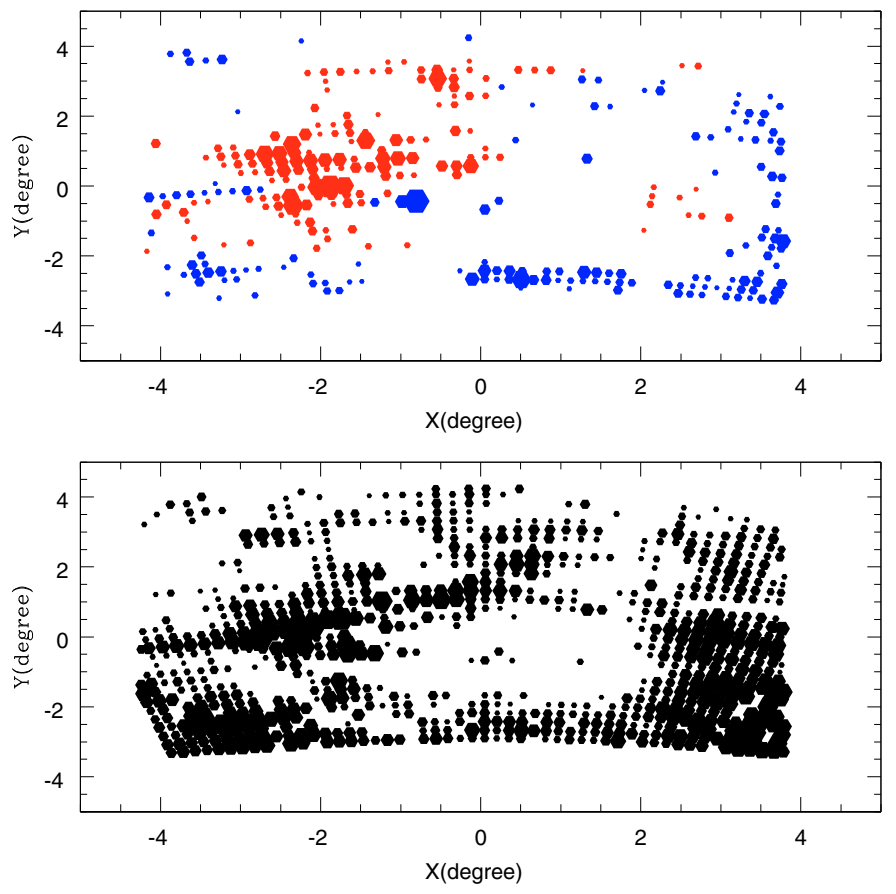

Fig. 5. Two-dimensional plot of the extinction, $A_{I}$ for the MCPS data is shown in the lower panel and a two-dimensional plot of deviation is shown in the upper panel. In the lower and upper panels the size of the points are proportional to the amplitude of the extinction and deviation of the regions from the plane of the LMC disk respectively. The blue and red points in the upper panel are the regions which are in front and behind the fitted plane respectively.

But again, to understand the effect of reddening on the detected extra-planar features, we plotted a two dimensional plot of reddening as well as the deviations. The plots for the MCPS and the OGLE III data are shown in Figs. 5 and 6 respectively. In both the plots the lower panel shows the reddening distribution and the upper panel shows the distribution of the deviations. In the lower panel the size of the point is proportional to the reddening value and in the upper plot the size of the point is proportional to the amplitude of the deviation. The red points in the upper panel of the plot represents the regions behind the plane and blue points represent the regions in front of the plane. We can see that in the MCPS plot (Fig. 5) the regions in the southwestern part of the LMC disk around our suggested warps show more reddening. Hence it is possible that these warps are due to overestimate of reddening in these regions. Olsen \& Salyk (2002) 
Table 2. Summary of orientation measurements of the LMC disk plane with choice of the center

\begin{tabular}{lcc}
\hline \hline Reference & Inclination, $i$ & $\mathrm{PA}_{\text {lon }}, \phi$ \\
\hline MCPS data & $37^{\circ} .4 \pm 2.3$ & $141^{\circ} .2 \pm 3^{\circ} .7$ \\
Optical center de Vaucouleurs \& Freeman (1972) & $37^{\circ} .2 \pm 2.2$ & $142^{\circ} \pm 3^{\circ} .4$ \\
HI rotation center (Kim et al. 1998) & $36^{\circ} .8 \pm 2.2$ & $141^{\circ} .4 \pm 3^{\circ} .8$ \\
Geometric center of the sample Cepheids used in the analysis of Nikolaev et al. (2004) & $36^{\circ} .9 \pm 2.5$ & $137^{\circ} .4 \pm 4^{\circ} .0$ \\
\hline Center of carbon stars outer isopleths (van der Marel \& Cioni 2001) & $23^{\circ} .0 \pm 0.8$ & $163^{\circ} .7 \pm 1^{\circ} .5$ \\
\hline OGLE III data & $23^{\circ} .1 \pm 0.8$ & $164^{\circ} .9 \pm 1^{\circ} .7$ \\
Optical center de Vaucouleurs \& Freeman (1972) & $22^{\circ} .7 \pm 0.8$ & $164^{\circ} .0 \pm 1^{\circ} .6$ \\
HI rotation center (Kim et al. 1998) & $22^{\circ} .0 \pm 0.8$ & $161^{\circ} .9 \pm 1.6$ \\
Geometric center of the sample Cepheids used in the analysis of Nikolaev et al. (2004) \\
Center of carbon stars outer isopleths (van der Marel \& Cioni 2001)
\end{tabular}
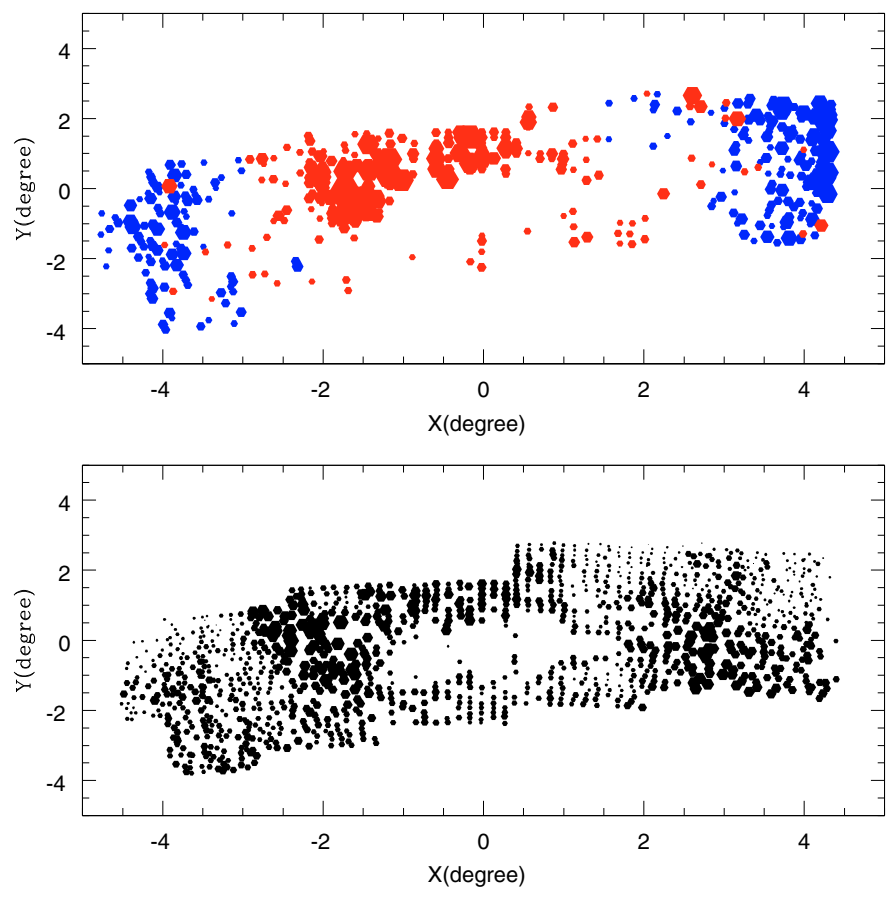

Fig. 6. Two-dimensional plot of the extinction, $A_{I}$ for the OGLE III data is shown in the lower panel and a two-dimensional plot of deviation is shown in the upper panel. In the lower and upper panels the size of the points are proportional to the amplitude of the extinction and deviation of the regions from the plane of the LMC disk respectively. The blue and red points in the upper panel are the regions which are in front and behind the fitted plane respectively.

also found that the reddening near the southwestern part of the disk near the regions of their suggested warps is stronger. They correlated the large reddening in the southwestern LMC regions to the diffuse extinction due to the high Galactic foreground dust, having $A_{I}$ of approximately $0.3 \mathrm{mag}$, given in the COBEDIRBE-IRAS/ISSA dust map (Schlegel et al. 1998). Olsen \& Salyk (2002) measured an $A_{I}$ of approximately 0.25 mag at the LMC's southwest edge. We measured an $A_{I}$ ranging from 0.1 to 0.2 with an average of $0.16 \mathrm{mag}$ at the LMC southwest edge. The plot which shows the amplitude of the deviation against the extinction values for the MCPS region is shown in Fig. 7. The magenta dots are the regions in the southwestern region of LMC, which are brighter than the surrounding regions. The reddening is high in these regions compared to the surrounding regions. There are also regions which are in front of the plane, like the southeastern disk, which do not show large reddening. Again, regions in the northeastern part with respect to the LMC bar show

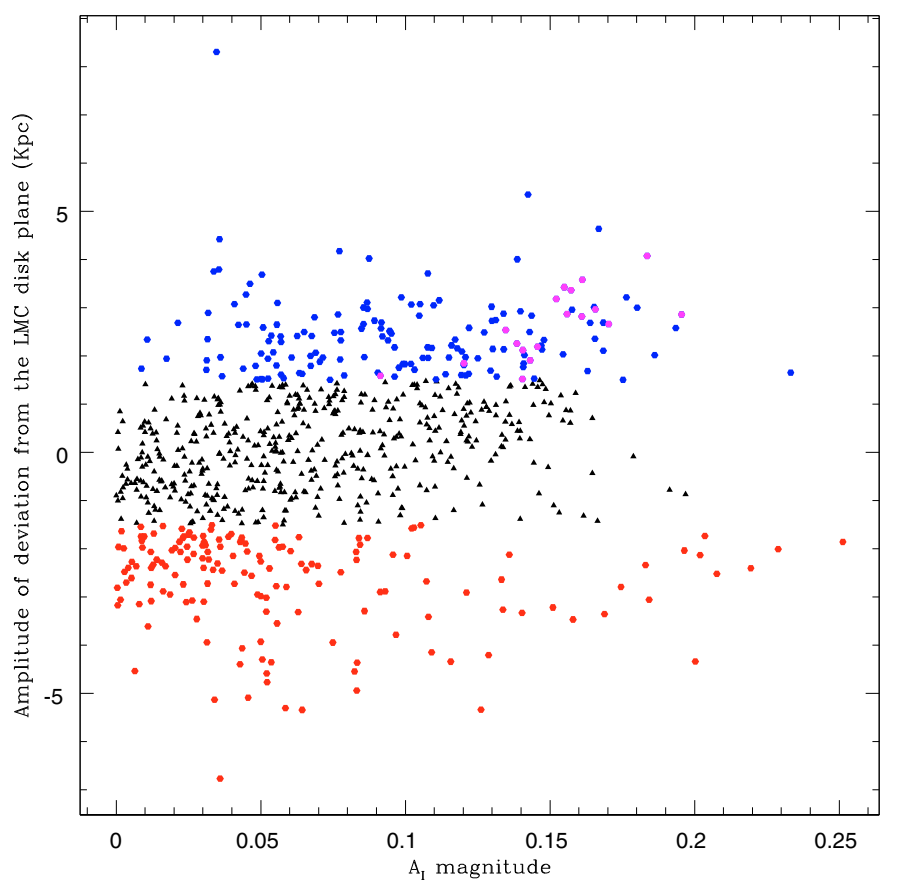

Fig. 7. Amplitude of the deviations of the regions from the fitted plane are plotted against the $A_{I}$ mag for the MCPS data. Black points are those with deviations less than 3 sigma, and red and blue are those with deviations more than 3 sigma and are behind the plane and in front of the plane respectively. Magenta dots are regions which are in front of the fitted plane in the southwestern part of the LMC disk.

large reddening and these regions are shown in the deviation plot as behind the fitted plane. Subramaniam et al. (2010, in preparation) show that these regions coincide with the star-forming regions. Once again, it could be argued that the reddening has not been accounted for properly here. There are also some nearby regions behind the fitted plane, which do not show large reddening. Thus some regions which are in the front or behind show a range of reddening as seen in Fig. 7. On the other hand, we do not see a strong correlation between reddening and the deviation, because both positive and negative deviations are observed for regions with large reddening. That is, the reddening could not have been both under and over-estimated. Thus, reddening is not strongly correlated with the estimated structures, but the extend of the deviation from the LMC plane may still be affected by the reddening that is present.

As mentioned in the analysis section, while estimating the reddening values for the MCPS regions, 451 regions out of 1231 regions showed a negative value for the reddening. We removed those regions from our analysis. Zaritsky et al. (2004) 


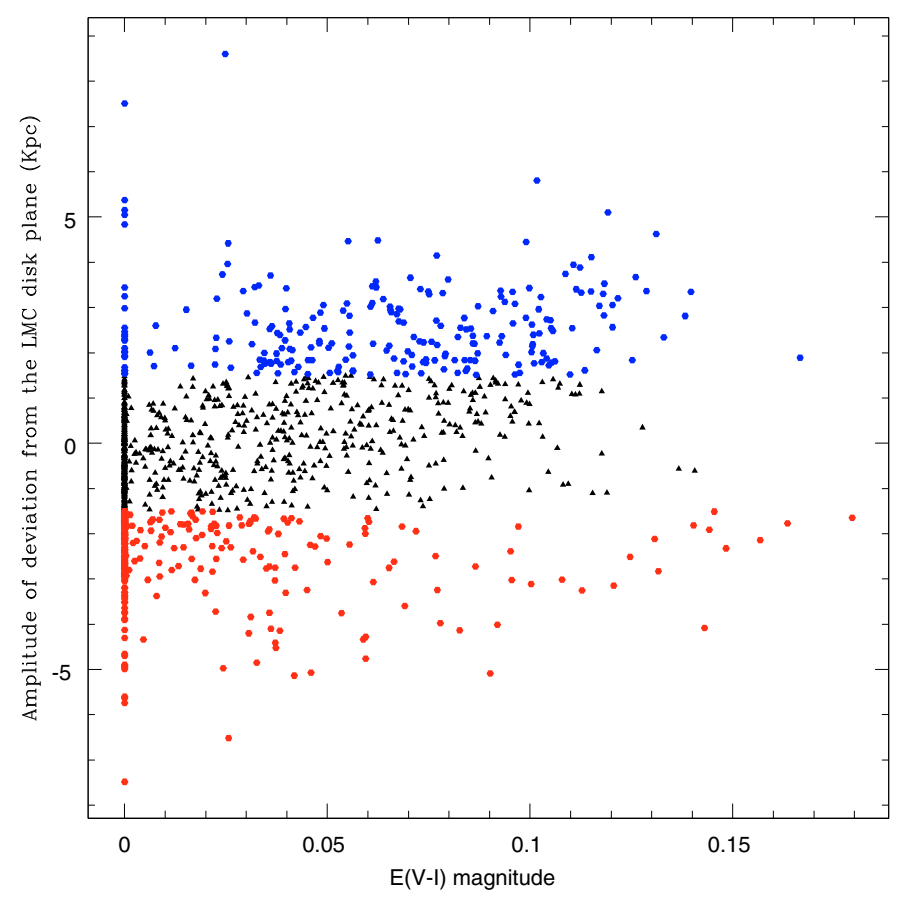

Fig. 8. Amplitude of the deviations of the regions from the plane of the LMC disk plane are plotted against the reddening, $E(V-I)$ values for the MCPS data after assigning zero reddening for the regions which show negative values for the reddening (see Fig. 7 for details).

estimated the extinction map for the LMC by comparing the stellar atmospheric models and observed colors using the MCPS data. They also found that many regions show negative extinction values. They suggested that large observational uncertainties scatters the extinction to negative values, and they set those negative values as zero extinction values. We estimated the structural parameters of the LMC disk using all the 1231 regions of the MCPS data after assigning zero reddening value for those regions which show negative value for the reddening. A weighted plane-fitting procedure was applied to the 1231 regions of the MCPS data and deviations were estimated. Deviations above 3 sigma were considered as deviations, and after removing those regions, planar parameters of the LMC disk plane was reestimated. An inclination, $i=39^{\circ} .3 \pm 3^{\circ} .2$ and $\mathrm{PA}_{\operatorname{lon}} \phi=139^{\circ} .0 \pm 4$ was obtained. The planar parameters are matching well with the parameters we estimated without considering the regions which showed negative values for reddening. To find where these regions are located with respect to the plane of the LMC disk, we plotted the reddening $E(V-I)$ mag vs. amplitude of the deviation calculated. This plot is shown in Fig. 8. We can clearly see that most of the regions which are assigned zero reddening values show large deviation from the plane. These regions are found both in front and behind the fitted plane. These deviations may be real. Because the reddening has an important role in the estimation of the structural parameters of the disk and the amplitude of the deviations, we prefer the analysis without considering regions with negative reddening. The agreement in the estimated parameters could just be coincidence because the deviations estimated are both in front and behind the plane and thus the net effect on the parameters of the LMC plane is minimum.

For the MCPS data in general the color of the RC stars is bluer when compared to the OGLE III RC stars. This can be due to the difference in the filter systems used and/or calibrations. The choice of intrinsic $(V-I)$ color of RC stars can be
Table 3. Summary of orientation measurements of the LMC disk plane at different radii.

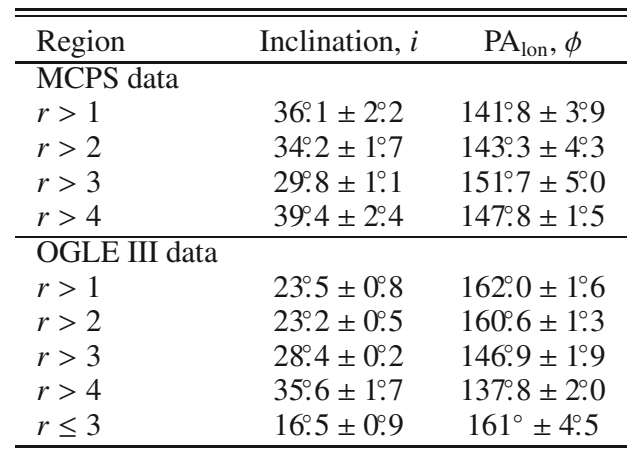

another reason for obtaining negative reddening values for a large number of regions in the MCPS data. The intrinsic color of RC stars for calculating reddening is taken to be $0.92 \mathrm{mag}$ (Olsen \& Salyk 2002). Olsen \& Salyk (2002) selected this value to produce the median reddening obtained toward the LMC by Schlegel et al. (1998). For the OGLE III data set we calculated the intrinsic $(V-I)$ color of RC stars to produce the median reddening obtained by Schlegel et al. (1998). The value turned out to be $0.915 \mathrm{mag}$, which is similar to the value given by Olsen $\&$ Salyk (2002). So we did the analysis of the OGLE III data with intrinsic color value, $0.92 \mathrm{mag}$. Also, there are no locations in the OGLE III data, where the value of estimated reddening goes negative. We calculated the intrinsic $(V-I)$ color of RC stars in the MCPS data set in a similar way as Olsen \& Salyk (2002). The value obtained is $0.84 \mathrm{mag}$. Using this value we repeated the analysis explained in Sect. 3 to estimate the LMC disk parameters. Now, out of 1231 locations, only 64 regions showed negative value for reddening. A plane fitting procedure is applied to 1167 regions and deviations from the plane are also estimated. The regions with deviations above 3 sigma are removed and the plane fitting procedure is applied to the remaining regions. The values obtained are $i=38^{\circ} .7 \pm 2.4$ and $\phi=150^{\circ} .9 \pm 3.8$. The inclination value is comparable and within errors with the analysis done with intrinsic $(V-I)$ color of RC stars, 0.92 mag. But the $\mathrm{PA}_{\text {lon }}$ value is higher than those obtained in the earlier analysis. Thus by including more regions in the inner $\mathrm{LMC}$, the $\mathrm{PA}_{\text {lon }}$ increased significantly, whereas the inclination did not change.

\subsection{Effect of coverage and inner structure of the LMC disk}

The estimated planar parameters of the LMC may vary depending on the difference in the coverage of the LMC studied to estimate these parameters. We also obtained significantly different results from the MCPS and the OGLE III data. In order to understand the effect due to coverage, we estimated the planar parameters of the LMC disk excluding the inner LMC data at different radii from the LMC center. Thus the planar parameters are estimated using the data above the radius of $1,2,3$, and 4 degrees from the LMC center. The results are summarised in Table 3. One of the important results of this analysis is that the parameters estimated excluding the data within the radius of 3 degrees from the LMC center in both the MCPS and the OGLE III sets are similar, as shown in Table 2. From Table 1 we can see that there is a significant difference in the estimates of the planar parameters, from the analysis of full data set of the OGLE III and the MCPS data. When the inner data are excluded from the analysis, the results are matching within the error bars. This indicates 
Table 4. Summary of orientation measurements of the LMC disk plane at different radial rings.

\begin{tabular}{lcc}
\hline \hline Region & Inclination, $i$ & $\mathrm{PA}_{\mathrm{Ion}}, \phi$ \\
\hline MCPS data & & \\
$2.5<r<3.5$ & $38^{\circ} 9 \pm 0.9$ & $161^{\circ} .9 \pm 3.5$ \\
$3.5<r<4.5$ & $25^{\circ} .2 \pm 1.4$ & $151^{\circ} .4 \pm 7.5$ \\
$4.5<r<5.5$ & $37.7 \pm 1.4$ & $134^{\circ} .2 \pm 0.7$ \\
\hline OGLE III data & & \\
$2.5<r<3.5$ & $21^{\circ} .4 \pm 0.3$ & $157^{\circ} .6 \pm 2.6$ \\
$3.5<r<4.5$ & $31^{\circ} .4 \pm 0.4$ & $139^{\circ} .5 \pm 1.4$ \\
$4.5<r<5.5$ & $39^{\circ} .1 \pm 1.6$ & $134.6 \pm 0.9$ \\
\hline
\end{tabular}

that the inner structure of the LMC disk affects the estimate of the LMC disk parameters. Even though both the MCPS and the OGLE III contain data within 3 degrees of the radius from the LMC center, the OGLE III data are more affected by the inner structures. This is because the OGLE III contains a highest number of inner regions compared to the MCPS data. As mentioned before, several central regions had to be removed from the analysis of the MCPS data because they showed negative reddening. That probably is the reason for a high $\mathrm{PA}_{\mathrm{lon}}$ and low inclination obtained from the OGLE III analysis.

To understand the inner structure of the LMC, we tried fitting a plane to the data within 3 degrees from the LMC center. For the MCPS data the fitting was a problem because of fewer points and also because of the deviations present within the 3 degree region from the center. For the OGLE III data the parameters obtained are $i=16.5 \pm 0.9$ and $\mathrm{PA}_{\text {lon }}=161^{\circ} \pm 4.5$. This indicates that the inner and outer structures of the LMC disk are different. Regions within the 3 degree radius fit to a plane with significantly less inclination and large $\mathrm{PA}_{\text {lon }}$, whereas regions outside 3 degrees have a large inclination and less $\mathrm{PA}_{\text {lon }}$.

A similar analysis was performed by van der Marel \& Cioni (2001), for AGB stars, where they estimated the variation of $i$ and $\mathrm{PA}_{\mathrm{lon}}$ as a function of radius. They used rings, whereas we used concentric regions progressively excluding the inner regions. To compare our results with theirs, we also obtained parameters of the LMC disk in different rings, and the results are given in Table 4. They found that the $i$ and $\mathrm{PA}_{\text {lon }}$ decrease with radius, whereas we find only the $\mathrm{PA}_{\text {lon }}$ to decrease with radius. Thus both analyses agree that the $\mathrm{PA}_{\text {lon }}$ decreases with increasing radius. The differences in the trend seen for the inclination may be due to the difference in the tracer and the method used. The inclination $i$ is found to be increasing with radius for the OGLE III data. For the MCPS data, we find that the outer two rings show an increasing $i$, whereas the innermost ring does not follow the trend. Therefore a definite pattern is not seen for the MCPS data set. Combining the result obtained in the last paragraph, we suggest that there is a difference in the structure of the LMC inside and outside a radius of around 3 degrees. Our results suggest that the outer disk is inclined more than the inner disk with a reduced $\mathrm{PA}_{\text {lon }}$. Subramaniam (2005a) proposed the existence of two disks in the inner $3^{\circ}$ of the LMC, with one counter rotating. May be the existence of two disks in the inner LMC is the reason that it is different from the outer structure.

\subsection{Deviations and warps}

After fitting the LMC plane, the deviations of the disk with respect to the fitted plane seen in the overlapping regions of the OGLE III and the MCPS data sets are similar. The extra-planar feature, which is seen as behind the LMC plane in the northeast
"IImcogle3xyznik.dat" u 1:2:7

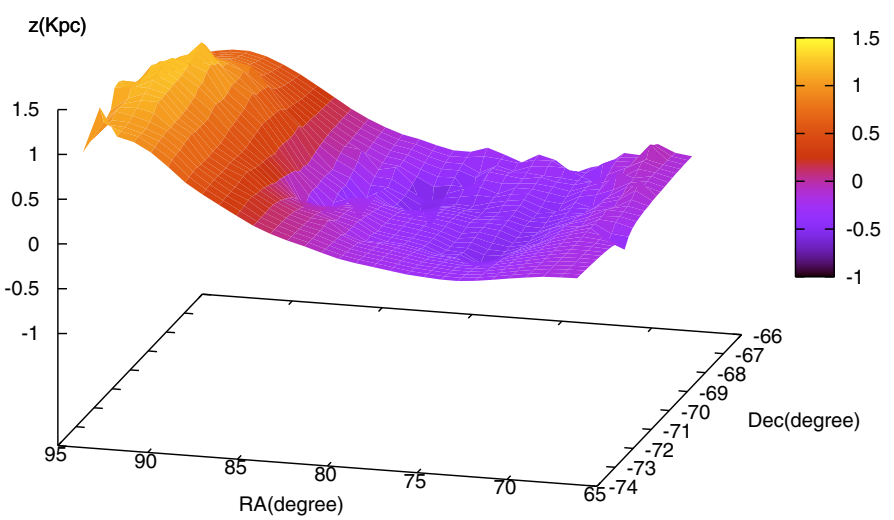

Fig. 9. 3D plot of the LMC disk obtained from the OGLE III RC stars.

of the LMC bar is interesting. This feature is seen in both the MCPS and the OGLE III deviation plots. An extension behind the disk in the same region is suggested by Subramaniam et al. (2009) in the study of RR Lyrae stars in the LMC. Some of the star-forming regions (Kim et al. 2000) are lying around this feature. Brightening of the RC stars in the western and eastern ends of the disk are seen in the OGLE III deviation plot. The brightening in the northwestern part of the LMC disk is more clearly seen with larger amplitude. The deviation of the the MCPS data shows a similar brightening in the northwestern, southwestern, and southeastern regions of the LMC disk. The warps suggested by Olsen \& Salyk (2002) in the southwestern region of the LMC are overplotted in our deviation plots, and not many regions which they suggest as warps are coinciding with our deviations, though they are located near the regions where we see deviations in the southwestern disk. Thus the brightening of the RC stars in the southwestern part of the LMC disk and the dimming of RC stars in the northeastern part near the LMC bar are suggestive of a symmetric warp in the LMC disk. Nikolaev et al. (2004) suggested a symmetric warp in the LMC disk similar to our results. Along with the symmetric warp, the brightening of RC stars in the northwestern part of the LMC disk is also very clearly seen in both the MCPS and the OGLE III deviation plots. The southeastern part of the LMC disk is also comparatively brighter than other regions, which makes the warp asymmetric. In the MCPS deviation plot, brightening of RC stars is seen near the optical center, in the bar region of the LMC. This kind of RC brightening is seen near the optical center in the bar region of the LMC by Subramaniam \& Subramanian (2009) and also by Koerwer (2009).

The edge-on view of the LMC as seen from the minor-axis (Fig. 4) also suggests that the inner LMC might have a different inclination with respect to the outer regions. Apart from this, the symmetric warp can also be noticed in this plot. It can also be noticed that the warp is not centered at the LMC center, but shifted towards the southwest by about a degree. Thus, our results suggest an off-centered symmetric warp. It is quite clear from the analysis that the inner structure of the LMC is quite complicated. In order to get a 3-D picture of the region studied here, we have shown the RA, Dec, and $Z$ plot of the OGLE III region in Fig. 9. The surface plot shown here is obtained with a standard surface plot algorithm and thus involves interpolation and averaging. Therefore, this plot can be used to obtain a qualitative understanding of the structure, but cannot be used 
for any quantitative analysis. The figure clearly shows that the inner regions have a smaller inclination and the outer regions have large inclination. The increase in the inclination seems to start at a closer radius in the northeast, when compared to the southwest, which makes it off-centered. It is clear from this figure that the LMC disk shows a lot of structures, and it is difficult to define a plane. By fitting a plane, a large number of regions are likely to show deviation from the plane suggesting warps. It is also clear that depending on the coverage of the data, the plane fitted and the parameters obtained are likely to change, which in turn will identify different regions to have deviations/warps. Also, defining a warp gets complicated due to contribution from reddening and population effects. In general, we notice that the inclination increases with radius. The northeast of the LMC is closer to us, and we find that it gets even closer with radius, with an increase in inclination. The southwest part, instead of getting more distant, also seems to get slightly closer. This is also seen as a warp by Olsen \& Salyk (2002). Thus, the plot suggests that studies which ignored the inner regions and considered only the outer regions are likely to derive a highly inclined plane with a smaller $\mathrm{PA}_{\text {lon. }}$. Studies that did not consider the outer regions would derive a less inclined plane with a large $\mathrm{PA}_{\text {lon }}$. Because the change in the inclination is off-centered, methods which use ring-analysis are likely to be more affected by the inner structure.

Warps and structural changes seen in the LMC disk could be due to tidal interactions. The Small Magellanic Cloud is unlikely to be the cause because it is smaller compared to the LMC. This effect may be due to the gravitational attraction of our Galaxy on the LMC. Thus, our results point in the direction that the increased inclination and the warps identified in the outer regions may be due to the interaction with our Galaxy. It is important to study more distant regions to understand the change in the disk structure with radius. A detailed study of the outer structure may throw light on the details of the tidal effects on the LMC disk and its origin.

\subsection{Comparison with the structure of the HI gas disk}

It is interesting to compare the structure of the HI gas disk of the LMC with that of the stellar disk. Various HI studies have estimated the $\mathrm{PA}_{\text {lon }}$ and the inclination of the HI disk by kinematical as well as by geometrical methods. These studies also suggested the presence of extra-planar features in the HI disk. Feitzinger et al. (1977) derived an inclination of $33^{\circ} \pm 3^{\circ}$ and $\mathrm{PA}_{\text {lon }}, 168^{\circ} \pm 4^{\circ}$ by geometrical means. The HI velocity studies by Luks \& Rohlfs (1992) revealed two kinematic components, the L (lower velocity) component and the D (disk) componenet. The D component was found to be extended in the whole LMC, and the L component, with two deformed lobes, was found to the north of 30 Dor and south of 30 Dor region. The L component was found to be around $50-500 \mathrm{pc}$ above the $\mathrm{D}$ component. The $\mathrm{PA}_{\text {lon }}$ of around $162^{\circ}$ was estimated for the disk component by kinematical method (line of maximum velocity gradient). The above values are not very different from the $\mathrm{PA}_{\text {lon }}$ of the stellar disk estimated from the OGLE III data. Kim et al. (1998) estimated the $\mathrm{PA}_{\text {lon }}$ of $\mathrm{HI}$ disk to be around $168^{\circ}$ by kinematical method. They suggested that the HI disk is inclined in a way that $\mathrm{PA}=78^{\circ}$ is closer to us. This value is agrees well with our result, which gives the PA of the closest part to be around $71^{\circ} \pm 4.5$ for the inner stellar disk from the OGLE III data. Kim et al. (1998) also estimated the inclination of the HI disk from the outer isophotes of HI brightness temperature to be around
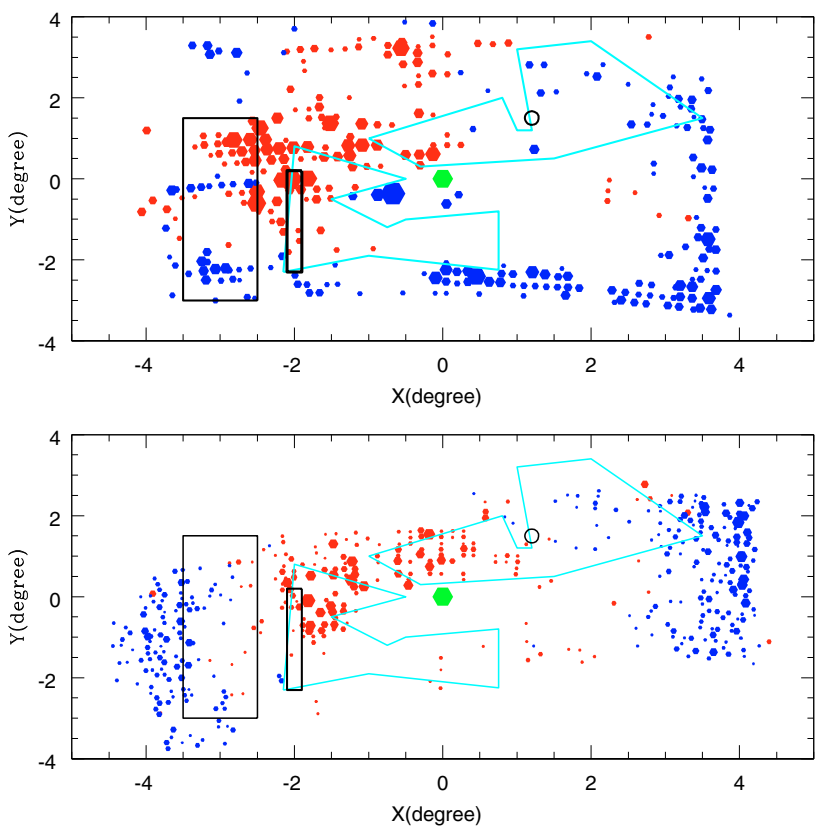

Fig. 10. Deviations found in the stellar disk of the LMC using the OGLE III and the MCPS data are plotted in the lower and upper panels respectively. The color code is the same as in Figs. 2 and 3. The two cyan lobes in both the panels are the L component identified in HI disk by Luks \& Rohlfs (1992). The black features in both the panels are the kinematical warps suggested by Luks \& Rohlfs (1992) in the L and D components.

$22^{\circ}$. As this value was poorly determined and highly correlated with other parameters, they adopted the canonical value of $33^{\circ}$ (Westerlund 1997).

In order to compare the HI and stellar disk structures, we plotted the deviations found in the stellar disk from the OGLE III and the MCPS data sets along with the structures found in the HI disk. In Fig. 10 the lower panel shows the deviations found in the OGLE III data and the upper panel shows the deviations found in the MCPS data set for the stellar disk. As in the previous plots, red points denote the regions behind the fitted plane and blue points denote the regions in front of the fitted plane, with sizes proportional to the amplitude of the deviations. The approximate location of the L component identified by Luks \& Rohlfs (1992) is shown as two irregular structures in cyan color. Luks \& Rohlfs (1992) suggested the presence of kinematical warps in some regions of the $\mathrm{D}$ component as well as in some regions of the L component. These regions are shown as black features. The kinematical warps identified in the D component are in the big rectangular box in the east. The regions where kinematical warps are identified in the L component are shown as black circle and small black rectangular box. Most of the kinematical warps identified in the HI disk are more or less near the extra-planar features found in the stellar disk.

The $\mathrm{PA}_{\text {lon }}$ estimated for the $\mathrm{HI}$ disk is similar to the $\mathrm{PA}_{\text {lon }}$ of the stellar disk in the inner regions of the LMC, particularly the estimates from the OGLE III data. The effect of inclination of the LMC, which makes the northeastern part appear to be closer, is seen both in stellar as well as in the HI disk of inner LMC. The inclinations are also similar. The kinematic warp seen in the $\mathrm{D}$ component coincides with the eastern stellar warp. There is a mild indication that the $\mathrm{L}$ component coincides with regions 
which are located behind the disk. On the whole, the L component and the kinematical warps identified in both components of HI, more or less coincide with the warps in the stellar disk. There is no HI counter part for the southwestern warp found in the stellar disk. These results suggest that the inner stellar and HI disk structures of the LMC are similar.

\section{Conclusions}

We used the RC stars identified from the OGLE III and the MCPS to estimate the structural parameters of the LMC. The results can be summarised as follows:

- We estimated the structural parameters of the LMC disk such as the inclination, $i$, and the position angle of the line of nodes $\left(\mathrm{PA}_{\text {lon }}\right), \phi$ using a weighted least-square plane-fitting procedure.

- We find an inclination of $i=23^{\circ} .0 \pm 0.8$ and $\mathrm{PA}_{\mathrm{lon}}, \phi=163^{\circ} .7 \pm$ 1.5 using the OGLE III data and an inclination of $i=37^{\circ} .4 \pm$ 2.3 and $\mathrm{PA}_{\text {lon }} \phi=141.2 \pm 3.7$ using the MCPS data. Extraplanar features, which are in front as well as behind the fitted plane, are seen in both the data sets.

- We find that the choice of center has a negligible effect on the estimated parameters.

- The reddening is found to be large in some regions located in front and also in regions located behind the fitted plane. Deviations are also found in regions without large reddening. These suggest that the reddening is only mildly correlated with the deviations of the disk from the fitted plane.

- We find that the disk within a 3 degree radius has lower inclination and higher $\mathrm{PA}_{\mathrm{lon}}$, and differs from the outer disk.

- The edge-on view of the LMC disk along the minor axis suggests an off-centered symmetric warp.

- The change of structure in the outer LMC could be due to tidal effects.

- We suggest that the complicated structure of the inner LMC causes variation in the estimated planar parameters depending on the area covered for each study, including the two data sets used here.
- In the inner LMC, the stellar as well as the HI disk have similar properties.

Acknowledgements. The authors thank the OGLE and the MCPS team for making the data available in public. We thank Knut Olsen for a critical reading of the manuscript and comments. We thank the referee for suggestions which improved the paper. Smitha Subramanian acknowledges the financial support provided by Council of Scientific and Industrial Research (CSIR), India through SRF grant, 09/890(0002)/2007-EMR-I.

\section{References}

Alves, D. R. 2004, New Astron. Rev., 48, 659

Caldwell, J. A. R., \& Coulson, I. M. 1986, MNRAS, 218, 223

de Vaucouleurs, G., \& Freeman, K. C., 1973, Vistas Astron., 14, 163

Feitzinger, J. V., Isserstedt, J., \& Schmidt-Kaler, Th. 1977, A\&A, 57, 265

Girardi, L., \& Salaris, M. 2001, MNRAS, 323, 109

Grocholski, A. J., Cole, A. A., Sarajedini, A., Geisler, D., \& Smith, V. V. 2006, AJ, 132, 1630

Holtzman, J. A., Gallagher, J. S. III., Cole, A. A., et al 1999, AJ, 118, 2262

Kim, S., Staveley-Smith, L., Dopita, M. A., et al. 1998, ApJ, 503, 674

Kim, S., Dopita, M. A., Staveley-Smith, L., \& Bessell, M. 2000, IAUJD, 1, 55

Koerwer, J. F., AJ, 138, 1

Luks, Th., \& Rohlfs, K. 1992, A\&A, 263, 41

Nikolaev, S., Drake, A. J., Keller, S. C., et al. 2004, ApJ, 601, 260

Olsen, K. A. G., \& Salyk.C. 2002, AJ, 124, 2045

Pagel B. E. J., \& Tautvaisiene G. 1998, MNRAS, 299, 535

Persson, S. E., Madore, B. F., Krzeminiski, W., et al. 2004, AJ, 128, 223

Salaris, M., \& Girardi, L. 2002, MNRAS, 337, 332

Schlegel, D. J., Finkbeiner, D. P., \& Davis, M. 1998, ApJ, 500, 525

Stanek, K. Z., Zaritsky, D., \& Harris, J. 1998, ApJ, 500, 141

Subramaniam, A. 2003, ApJ, 598, L19

Subramaniam, A. 2005a, ApJ , 625, L47

Subramaniam, A. 2005b, A\&A, 430, 421

Subramaniam, A., \& Anupama, G. C. 2002, A\&A, 390, 449

Subramaniam, A., \& Subramanian, S. 2009a, ApJ, 703, L37

Subramanian, A., \& Subramaniam, S. 2009b, A\&A, 503, L9

Subramanian, S., \& Subramaniam, A. 2009c, A\&A, 496, 399

Udalski, A., Soszynski, I., \& Szymanski, M. 2008, Acta Astron., 58, 89

van der Marel, R. P., \& Cioni, M. L. 2001, AJ, 122, 1807

Weinberg, M. D., \& Nikolaev, S 2001, ApJ, 548, 712

Westerlund, B. E. 1997, The Magellanic Clouds, Cambridge (Cambridge Univ. Press)

Zaritsky, D., Harris, J., \& Thompson, I. 1997, AJ, 114, 1003

Zaritsky, D., Harris, J., Thompson, I. B., et al. 2004, AJ, 128, 1606 